\title{
'GIVE IT TO ME'/ 'GIVE IT TO HER': THE DEVELOPMENT OF A RELATIONAL TERM IN HOMESIGN AND ENGLISH
}

\author{
Catriona Silvey $^{* 1}$ and Susan Goldin-Meadow ${ }^{2}$ \\ *Corresponding Author: c.silvey@ucl.ac.uk \\ ${ }^{1}$ Division of Psychology and Language Sciences, University College London, London, UK \\ ${ }^{2}$ Departments of Psychology and Comparative Human Development, University of Chicago, \\ Chicago, USA
}

In typical language acquisition, evidence from naturalistic and experimental studies suggests that the meanings of relational terms such as verbs are harder for children to learn than the meanings of nouns (Gentner, 1982; Gleitman, Cassidy, Nappa, Papafragou, \& Trueswell, 2005). Children's eventual acquisition of verb meanings is explained as being supported by other aspects of the linguistic system. By repeatedly highlighting relations across contexts (Loewenstein \& Gentner, 2005) or bootstrapping meaning through syntax (Gleitman et al., 2005), the structure of language as a whole is argued to be crucial to children's acquisition of relational terms. However, from a language evolution perspective, this explanation is not entirely satisfactory: if a fully developed linguistic system is required to learn these meanings, then what is their evolutionary origin?

One source of evidence to address this question is homesign. Homesigners are deaf children raised by hearing parents who do not expose them to a sign language. These children are unable to access spoken language input, and their parents' gestural input is unsystematic; however, the children spontaneously invent gestural systems for communication, referred to as homesign. These systems have many of the features of natural languages (Goldin-Meadow, 2003), including a distinction between nouns and verbs (Goldin-Meadow, Butcher, Mylander, \& Dodge, 1994). Homesign is a crucial and hitherto overlooked source of evidence in the debate about the acquisition of relational terms. By examining the similarities and differences between the parent's gestural input and the child's homesign, we can determine which relational concepts are developed and generalized on the basis of evidence from input, versus factors internal to the child.

We focus here on the relational concept lexicalized by the English word 'give', and specifically on the role of the recipient. We compare the expression of this concept in the productions of one homesigner, David, observed over 4 sessions from the ages of 34 to 39 months, and of 15 typically developing children acquiring English, observed over 12 sessions from the ages of 14 to 58 months. 
From his first session, David produces a gesture (GIVE), which has a stable form (Goldin-Meadow et al., 1994) and, like the English verb 'give', appears to be a three-place predicate involving an agent, a patient and a recipient (GoldinMeadow et al., 1994). Initially, during the 3 sessions from 34 to 36 months, the recipients in David's uses of GIVE are restricted to himself (47 tokens) or locations adjacent to him ( 2 tokens). Only later, in the 39 month session, does he use GIVE with another person as a recipient ( 2 of 9 tokens). There are two potential explanations for this change. One is that it is due to maturational or motivational factors. Another possibility is that this an effect of input: during the previous session, when David was 36 months old, his mother gestured GIVE and specified David's sister as a recipient. This sequence is compatible with the hypothesis that David's GIVE gesture initially has a meaning closer to 'give-to-me/put-near-me', which he then generalizes on the basis of maternal input that suggests a broader range of uses. However, before drawing this conclusion, it is crucial to look at children exposed to typical natural language input for comparison.

We analyzed early productions of 'give' by 15 typically developing children acquiring English. During the first observational session in which they produced 'give' (average 3 tokens), all but 2 of the 15 children used it in a similarly restricted way to David, i.e., with only the self as recipient. All but 1 child later produced 'give' with other recipients in subsequent sessions. To determine whether variation in children's onset of using 'give' with other recipients could be explained by input, we analyzed parents' use of 'give' (average 22 tokens) prior to each child's first production of the verb. All parents produced 'give' with other recipients during sessions prior to their child's first production of the verb. Thus, children's uses of 'give' are initially restricted despite evidence for generalization. Furthermore, the amount or proportion of parental uses of 'give' with other recipients does not predict children's age at first using 'give' with other recipients, suggesting that input is not the decisive factor for children's productive generalization; motivational or other factors may be involved. Indeed, David's age of onset of GIVE with other recipients (39 months) falls close to the median age of onset in English-acquiring children (38 months). Previous work showed that children's verbs encode their own actions before they encode the actions of others (Huttenlocher, Smiley, \& Charney, 1983). Our results suggest that rather than always focusing on the self as actor, children may initially use the role most salient to them (recipient, in the case of GIVE) as a 'way in' to the argument structure of a verb.

The factors that influence the development of GIVE/'give' in production may be relatively independent of language input. However, other relational terms, such as mental state verbs, are notably absent from homesign. This suggests an evolutionary story where some relational terms can develop without language input, whereas others need the support of a full linguistic system. Future work should investigate the factors that determine which relational terms fall into which category, and the processes by which more language-dependent relational terms emerge. 


\section{References}

Gentner, D. (1982). Why nouns are learned before verbs: Linguistic relativity versus natural partitioning. In Language development, volume 2: Language, thought and culture (pp. 301-334). Hillsdale, NJ: Lawrence Erlbaum.

Gleitman, L. R., Cassidy, K., Nappa, R., Papafragou, A., \& Trueswell, J. C. (2005). Hard words. Language Learning and Development, 1(1), 23-64.

Goldin-Meadow, S. (2003). The resilience of language: What gesture creation in deaf children can tell us about how all children learn language. New York: Psychology Press.

Goldin-Meadow, S., Butcher, C., Mylander, C., \& Dodge, M. (1994). Nouns and verbs in a self-styled gesture system: what's in a name? Cognitive Psychology, 27, 259-319.

Huttenlocher, J., Smiley, P., \& Charney, R. (1983). Emergence of action categories in the child: Evidence from verb meanings. Psychological Review, 90(1), 72-93.

Loewenstein, J., \& Gentner, D. (2005). Relational language and the development of relational mapping. Cognitive Psychology, 50(4), 315-53. 